\title{
CAPECITABINE AND IRINOTECAN AS FIRST- LINE CHEMOTHERAPY FOR PATIENTS WITH METASTATIC COLORECTAL CANCER
}

\author{
Deyan Davidov, \\ Department of Chemotherapy, Oncological Center, \\ Medical University, Pleven, Bulgaria
}

\section{RESUME:}

Objectives: Capecitabine is an oral fluoropyrimidine carbamate that is at least as effective than Fluorouracil / Leucovorin as first- line treatment for patients with metastatic colorectal cancer /CRC/. The topoisomerase I inhibitor Irinotecan has shown consistent efficacy in chemotherapynaive patients. The aim of this study was to determine the efficacy and tolerability of Capecitabine in combination with Irinotecan /XELIRI/ as first- line therapy in patients with advanced CRC. Methods: In the period 2007- 2009 twentyone consecutive patients with metastatic CRC entered the study. The treatment schedule consists of Capecitabine 1250 $\mathrm{mg} / \mathrm{m} 2$ p.o. twice daily for 14 days with a 7 - day rest period and intravenous Irinotecan $180 \mathrm{mg} / \mathrm{m} 2$ day 1 with repetition of courses every 21 days. Results: Overall response rate was $33,3 \%$. Median time to progression and overall survival was 7,6 months and 15,6 months, respectively. The most common grade 3- 4 adverse events were diarrhea and neutropenia. There were no treatment- related deaths. Conclusion: These results indicate that XELIRI is a potentially feasible and clinically active regimen in patients with advanced CRC.

Key words: Metastatic colorectal cancer, Capecitabine, Irinotecan, First- line chemotherapy, Survival

\section{INTRODUCTION}

Colorectal cancer /CRC/ is one of the most frequently encountered malignancies, third behind breast and cervical cancer in women and fourth after lung, stomach, and prostate cancer in men. Approximately 780000 new cases are diagnosed every year throughout the word and the estimated annual death rate is $440000 / 1 /$. Early- stage CRC is localized and resectable, but up to $30 \%$ of the patients already have metastatic disease at the time of diagnosis, and $20 \%-50 \%$ of patients with stage II and III will progress to stage IV. Approximately $50 \%$ to $60 \%$ of all patients die of progression of the disease. The five- years survival rate for stage IV disease overall, remains approximately of $10 \% / 2 /$.

Chemotherapy is effective in prolonging survival and time to disease progression in patients with metastatic CRC. Until recently, the therapeutic options for metastatic CRC were mainly confined to chemotherapy with 5-Fluorouracil /
$\mathrm{FU} /$, which was developed more than 50 years ago and is included in almost all standard and experimental regimens of palliative chemotherapy for $\mathrm{CRC} / 3 /$. A fluorinated pyrymidine, FU acts by inhibiting thymidylate synthase, an enzyme necessary for the production of thymidine nucleotides required for DNA synthesis. Numerous attempts have been made to improve the efficacy of FU, including biomodulation and schedule modification. Protracted infusion of FU and biomodulation with agents such as Leucovorin /LV/ have both resulted in improved response rates compared with bolus FU alone, but neither approach has demonstrated a clinically meaningful benefit in randomized trials or meta- analyses $/ 4 /$.

The recent development of new cytotoxic drugs and alternatives to FU with substantial antitumoral activity in metastatic CRC has dramatically changed treatment strategies and therapeutic goals in patients with advanced disease. In the 1990s, two additional agents, Irinotecan and Oxaliplatin, was found to have activity against advanced CRC. Irinotecan, a topoisomerase I inhibitor, offers mechanism of action completely different from those of FU in the treatment of CRC. Irinotecan and its metabolites bind to a complex of DNA and topoisomerase I - an enzyme required for unwinding of DNA during replication, inducting DNA strand breaks and consequent tumor death $/ 5 /$. Irinotecan has shown consistent efficacy in both chemotherapy- naive and 5- FUpretreated patients with metastatic CRC. The most frequent adverse events associated with Irinotecan are neuthropenia, delayed diarrhea, acute cholinergic syndrome and nausea/ vomiting.

An alternative approach to optimizing FU- based therapy has been the development of oral fluoropyrimidine derivates designed to deliver FU to the target cells. Oral administration enables sustained exposure to FU, avoids the technical barriers of intravenous administration and allows significant flexibility in the choice of the dosage regimen. In addition, most patients prefer oral cytotoxic therapy to intravenous regimens, provided that efficacy is not compromised $/ 6 /$.

Capecitabine, an oral fluoropyrimidine carbamate, was rationally designed to generate FU predominantly within tumor cells $/ 7 /$. After rapid and extensive absorption as an intact molecule, capecitabine is converted to 5- FU 
predominantly in tumor tissue by exploiting the high activity of thymidine phosphorylase in malignant tissue $/ 8 /$. The enzymatic conversion of capecitabine occurs in three steps. In the first stage, capecitabine is hydrolyzed by hepatic carboxylesterase to 5'-deoxy-5-fluorocytidine. This intermediate is then converted to 5'-deoxy-5-fluorouridine / 5 '-DFUR/ by cytidine- deaminase in tumor cells and the liver. The third and final step involves the conversion of 5'-DFUR to FU by thymidine phosphorylase and occurs predominantly in tumor tissue as result of the high activity of thymidine phosphorylase /9/. The increasing specificity for tumor cells occurring with each successive conversion step potentially reduces systemic 5-FU exposure while increasing the 5-FU dose within tumor tissue. The tumor selectivity of Capecitabine has been confirmed in patients with colorectal cancer. Patients received Capecitabine $1250 \mathrm{mg} / \mathrm{m} 2$ twice daily for 5 to 7 days before surgical resection of their primary tumor and/or liver metastases. Concentrations of FU in primary tumor tissue were 3,2- fold higher than in adjacent healthy tissue and 21-f old higher than in plasma /10/.

Since Capecitabine has proven efficacy in both the metastatic and adjuvant settings and simplifies administration of combination regimens as a result of its 3-weekly schedule, the logical step was to investigate the combination of Capecitabine and Irinotecan as first- line therapy in patients with advanced CRC.

Combining Irinotecan with oral Capecitabine is compelling because the afents have different modes of action, only partially overlapping adverse event profiles and administrtion of oral home- based Capecitabine is convenient. Synergistic antitumor activity has been observed with Capecitabine and Irinotecan in various xenograft models. This early promise of adding Capecitabine to Irinotecan in the preclinical setting has since been confirmed by encouraging results from phase I and II studies of Capecitabine in combinations with either weekly Irinotecan /CAPIRI schedule/ or 3-weekly Irinotecan /XELIRI schedule/ as first line therapy for patients with metastatic CRC. No significant pharmacokinetic interactions have been reported with these combinations / $11 /$.

The aim of this study was to determine the efficacy and tolerability of Capecitabine in combination with Irinotecan as first- line therapy in patients with advanced CRC.

\section{PATIENTS AND METHODS}

Twenty- one consecutive patients with metastatic CRC, treated in the period 2007- 2009 in Medical University- Pleven, Oncological center, Department of Chemotherapy, entered the study. To be eligible for treatment, participants had to be: between 18 and 75 years of age; histologically documented adenocarcinoma of the colon or rectum; progressive measurable metastatic disease; life expectancy of minimum three months; World Health Organisation /WHO/ performance status 0 to 2; no prior chemotherapy or radyotherapy for metastatic disease; adequate bone marrow function /absolute granulocyte count $>1,5 \times 10^{9} / \mathrm{L}$, platelet count $>140 \times 10^{9} / \mathrm{L} /$ as well as normal renal /serum creatinine level $<1,5 \mathrm{mmol} / \mathrm{L} /$ and hepatic function /serum bilirubin level $<21 \mathrm{mmol} / \mathrm{L} /$; absence of active infections; no overt cardiac disease. Measurable disease was assessed by computed tomography scan. This study required that previous adjuvant FU- based therapy be completed at least 6 months prior start of treatment.

The following was excluded: pregnant patients; women of childbearing potential who were not using reliable contraception; patients with central nervous system metastases, bowel obstruction or ileus; other malignancy; inability of swallow pills; major surgery within 4 weeks prior to treatment start. Capecitabine was administered at a dose of $1250 \mathrm{mg} / \mathrm{m} 2$ twice daily as an intermittent regimen in 3- week cycles- 2 weeks of treatment followed by 1 week of rest. Capecitabine was given at approximately 12 hours intervals orally with water within 30 min of ingesting food. Irinotecan was administered as an intravenous infusion over $90 \mathrm{~min}$ at a dose of $180 \mathrm{mg} / \mathrm{m} 2$ at day 1 . Treatments were repeated every 3 weeks up to six cycles and was stopped in the event of disease progression, serious adverse events or request by the patient. All patients received pre- medication with antiemetic drugs/ e.g. 5- HT antagonists before intravenous administration of Irinotecan. Administration of Capecitabine was interrupted if diarrhea grade $>1$ loccurred or if grade 1 toxicity persisted for more than two days. Furthermore, all patients were instructed than in case of diarrhea they have to take $4 \mathrm{mg}$ Loperamide orally immediately, and to continue with a dose of $2 \mathrm{mg}$ every 2 hours for at least 12 hours. If diarrhea persisted for $>24$ hours, oral antibiotics /usually Ciprofloxacin/ were administered additionally.

Patients were evaluated for tumor response before treatment and after every two courses of chemotherapy. Tumor response was evaluated according to WHO response criteria /12/. Response was defined as complete response / $\mathrm{CR} /$, partial response $/ \mathrm{PR} /$, no change $/ \mathrm{NC} /$, or progressive disease /PD/. A CR was defined by the disappearance of all known disease, confirmed by two observations not less than 4 weeks apart. PR was defined as a decrease in tumor size of $50 \%$ or more /either measured or estimated in the case of measurable or assessable disease/. In addition, there could be no appearance of any new lesions or progression of any known lesion. Objective tumor response included both confirmed CR and PR. Safety was assessed using the WHO toxicity criteria $/ 13 /$.

The duration of response was calculated from the day of the start of treatment to disease progression; overall survival was measured from study entry to death. The time to disease progression was calculated from study entry until the day of the first evidence of disease progression. The actuarial survival was estimated by the method of Kaplan and Meyer /14/. 


\section{RESULTS}

\section{Patients and Treatment}

A total of 21 patients were recruited in the study over a 24- months period. Data were collected for an additional 12 months after accrual ended, with data on survival collected through February 2010. All patients regardless of their length of treatment were included in analysis. Tumor response was evaluated for all patients who received at least one dose of XELIRI. All patients regardless of their length of treatment were included in analysis. Baseline demographic and disease characteristics are summarized in Table 1. The median age of patients were 58,9 years /range $43-74$ years/ and $32 \%$ of patients were $>65$ years. The male/ female ratio was $76,2 \%$ to $23,8 \%$. Median WHO- performance status score was $1 /$ range $0-2 /$. The majority of patients had rectum cancer$57,2 \%$. All patients had advanced or metastatic disease and the most frequently metastatic site were liver $/ 66,6 \% /$. As previous therapy, surgery only was used in 9 patients $/ 42,8 \% /$ , surgery and radiotherapy in 4patients $/ 19,0 \% /$ and surgery and adjuvant chemotherapy with FU/ LV was used in 8 patients $/ 38,2 \% /$. Median duration of treatment was 6,5 months. The follow- up period was 18 months.

\section{Antitumor effects}

The resulting antitumor effects are presented in Table 2. All 21 patients were evaluable for response. The overall response rate /ORR/ was 33,3\%/7 of 21 patients/ including two complete and five partial remissions. The median duration of response was 8,4 months. Median time to disease progression was 7,6 months. Overall survival was 15,6 months. Regression analysis identified poor WHO performance status, multiple site of metastases and liver as predominant site of metastases as prognostic factors, correlated with reduced overall survival.

\section{Safety}

The safety was assessed in all 21 patients. Most adverse events were mild to moderate in intensity and manageable through either temporally dose interruption or reduction. The most common hematological and nonhematological adverse drug reactions grade 3 and 4 that occurred in entire group are presented in Table 3 and Table 4. The highest incidence was gastrointestinal and hematology. Grade 3- 4 gastrointestinal and hematology toxicity was observed in $17,6 \%$ of the patients. There were no treatmentrelated deaths. The most common reasons for dosemodification of Irinotecan was neuthropenia and for Capecitabine was diarrhea. These adverse reactions were usually managed with Granulocyte colony- stimulating factors or Loperamide and never was fatal.

\section{DISCUSSION}

In the current study we evaluated efficacy and safety of the Capecitabine in combination with Irinotecan as first- line therapy in patients with advanced CRC. Our results indicate that XELIRY is an effective regimen. Tumor control / $\mathrm{CR}+\mathrm{PR}+\mathrm{NC} /$ was achieved in 15 patients $/ 71,4 \% /$. The overall response rate- $33,3 \%$, median time to disease progression- 7,6 months and overall survival- 16,5 months are similar to results previously reported with Irinotecan and infusional FU/ LV combinations and are also consistent with those from a studies, evaluating XELIRI regimens/15/. In these studies response rate were $21 \%-43 \%$ with time to disease progression of 5 - 8 months.

In the majority of patients the chemotherapy regimen was well tolerated. Both hematological and nonhematological toxicity was mild to moderate and chemotherapy was not stopped because of toxicity. Gastrointestinal toxicity or stomatitis never was fatal. Diarrhea, a well-known side effect of Capecitabine was generally managed with loperamide, which was administered to approximately one third of patients.

In conclusion, the results of the present study indicate that the treatment with Capecitabine in combination with Irinotecan as first- line therapy in patients with advanced CRC appears promising with of survival rate of 15,6 months and moderate toxicity.

Table 1. Patient characteristics

\begin{tabular}{|l|r|}
\hline Patient characteristics & Number of patients \\
\hline Age (years) & $43-74$ \\
\hline Sex & \\
Males & $16(76,2 \%)$ \\
Females & $5(23,8 \%)$ \\
\hline Performance status /WHO scale/ & \\
0 & $4(19,0 \%)$ \\
1 & $12(57,1 \%)$ \\
2 & $5(23,9 \%)$ \\
\hline Location of primary tumor & \\
Colon & $9(42,8 \%)$ \\
Rectum & $12(57,2 \%)$ \\
\hline Metastatic sites & \\
Liver & $14(66,6 \%)$ \\
Lung & $5(23,8 \%)$ \\
Peritoneum & $2(9,6 \%)$ \\
\hline N of metastatic sites & \\
1 & $16(76,2 \%)$ \\
2 & $3(14,3 \%)$ \\
3 & $2(9,5 \%)$ \\
\hline Previous treatment & \\
Surgery & $9(42,8 \%)$ \\
Surgery+ radiotherapy & $4(19,0 \%)$ \\
Surgery+ chemotherapy & $8(38,2 \%)$ \\
\hline
\end{tabular}


Table 2. Objective responses

\begin{tabular}{|l|c|c|c|c|c|}
\hline $\begin{array}{l}\text { Patients/ } \\
\text { Response }\end{array}$ & CR & PR & NC & PD & ORR\% \\
\hline 21 & 2 & 5 & 8 & 6 & $33,3 \%$ \\
\hline
\end{tabular}

$\mathrm{ORR}=\mathrm{CR}+\mathrm{PR}$

CR, Complete response; PR, Partial response; NC, No change; PD, Progressive disease; ORR, Overall objective response rates;

Table 3. Adverse drug reactions by symptoms: grade 3 and 4 hematological toxicity

\begin{tabular}{|l|r|}
\hline Adverse drug reactions & Number of patients \\
\hline Leukopenia & $2(11,7 \%)$ \\
Thrombocytopenia & $1(5,9 \%)$ \\
Anemia & $1(5,9 \%)$ \\
\hline
\end{tabular}

Table 4. Adverse drug reactions by symptoms: grade 3 and 4 non-hematological toxicity

\begin{tabular}{|l|r|}
\hline Adverse drug reactions & Number of patients \\
\hline Nausea & $3(17,6 \%)$ \\
Vomiting & $2(11,7 \%)$ \\
Diarrhea & $3(17,6 \%)$ \\
Stomatitis & $2(11,7 \%)$ \\
HFS & $1(5,9 \%)$ \\
Alopecia & $1(5,9 \%)$ \\
\hline
\end{tabular}

\section{REFERENCES:}

1. Ferlay J, Tiwari RC, Murray T. et al, Cancer Statistics, 2004. CA Cancer J Clin, 2004; 54: 8-29

2. Benson AB 3rd: Therapy for advanced colorectal cancer. Semin Oncol, 1998 Oct; 25(5 Suppl 11):2-11

3. Meta-analysis group in cancer. Efficacy of intravenous continuous infusion of fluorouracil compared with bolus administration in advanced colorectal cancer. J Clin Oncol, 1998 Jan;16(1):301-308

4. Thirion P., Wolmark N, Haddad E. et al, Survival impact of chemotherapy in patients with colorectal metastases confined to the liver: a re- analyses of 1458 nonoperable patients randomized in 22 trials and 4 meta- analyses. Meta- analysis group in cancer. Ann Oncol, 1999 Nov; 10(11):13171320

5. Pommier Y, Tanizava A, Kohn KW. Mechanisms of topoisomerase I inhibition by anticancer drugs. In: Liu LF, ed. Advanced in pharmacology. New York: Academic Press 1994; 29B; 73-92
6. Meropol NJ: Oral fluoropyrimidines in the treatment of colorectal cancer. Eur J Cancer, 1998; 34:1509-1513

7. Ishikawa T, Utoh M, Sawada N, et al: Tumor selective delivery of 5fluorouracil by capecitabine, a new oral fluoropyrimidine carbamate, in human cancer xenografts. Biochem Pharmacol, 1998; 55:1091-1097

8. Miwa M, Ura M, Nishida M, et al: Design of a novel oral fluoropyrimidine carbamate, capecitabine, which generates 5fluorouracil selectively in tumours by enzymes concentrated in human liver and cancer tissue. Eur J Cancer, 1998; 34:12741281

9. Mackean M, Planting A, Twelves C, et al: Phase I and pharmacologic study of intermittent twice-daily oral therapy with capecitabine in patients with advanced and/ or metastatic cancer. J Clin Oncol, 1998 Sep;16(9):2977-2985

10. Schuller J, Cassidy J, Dumont E, et al: Preferential activation of capecitabine in tumor following oral administration in colorectal cancer patients. Cancer Chemother Pharmacol, 2000 45:291-297

11. Delord JP, Pierga JY, Dieras F. et al, A phase I clinical and pharmacokinetic study of capecitabine /Xeloda/ and irinotecan combination therapy /XELIRI/ in patients with gastrointestinal tumours, Br J Cancer, 2005 Mar;92(5): 820- 826

12. Miller AB, Hoogstraten B, Staquet $M$ et al, Reporting results of cancer treatment. Cancer 1981; 47; 207-214

13. Brimdage MD, Pater JL, Zee B, Assessing the reliability of two toxicity scales: Implications for interpreting toxicity data. J Natl Cancer Inst 1993; 85; 38-48

14. Kaplan EL, Meyer P, Nonparametric estimation from incomplete observations, J Am Stat Assoc 1959; 53; 457- 481

15. Gill Sharlene, Treatment of metastatic colorectal cancer, Oncol Exch, 2005; 5: 6- 9

\author{
Address for correspondence: \\ Dr. Deyan Davidov, \\ Department of Chemotherapy, Oncological Center, Medical University, \\ 1 "St. Kliment Ohridsky" Str., 5000 Pleven, Bulgaria \\ Phone: +359/64/886 317, Fax: +359/64/831634 \\ E-mail: dean_davidov@abv.bg
}

